\title{
Developmental Dyslexia Studies in Chinese Learners
}

\author{
Li Guo \\ Beijing Language and Culture University, Beijing, China \\ *Corresponding author: hyguoli@163.com
}

Received April 25, 2014; Revised May 18, 2014; Accepted May 18, 2014

\begin{abstract}
The developmental dyslexia studies in alphabetic languages usually from the perspectives of cognition, neurobiology and genetics. Since the Chinese language is characterized as a logographic writing system with deep orthography, which is different from alphabetic languages, whether the findings in alphabetic languages are coincident in Chinese remains a question. By comparing theories in the causes of developmental dyslexia in alphabetic language with that in Chinese language, the paper reviews the studies on development dyslexia in L1 Chinese learners and finally reach the conclusion that although developmental dyslexia in Chinese language have been studied from cognition, neurobiology and genetics, which is coincident with those of alphabetic languages, there exits some differences in minor parts of the theories.
\end{abstract}

Keywords: Dyslexia, Chinese, Cognition, Neurobiology, Genetics

Cite This Article: Li Guo, "Developmental Dyslexia Studies in Chinese Learners.” Research in Psychology and Behavioral Sciences, vol. 2, no. 2 (2014): 43-47. doi: 10.12691/rpbs-2-2-2.

\section{Introduction}

Developmental Dyslexia (DD), which is known as specific reading disability, is a complete cognitive disorder [1]. The children with dyslexia will find it difficult to read although they are normal in IQ, equal in educational opportunities and absent in emotional disorders. And usually the individuals with developmental dyslexia have difficulties with accurate or fluent word recognition and spelling despite adequate instruction and intelligence and intact sensory abilities. Although the definition of DD varies greatly in different researchers and institutions, they all reach the agreement that DD is a language-based learning disability which caused by neuron-developmental disorder with a probable genetic bases [2].

Study in DD originates in 1880s with Oswald Berkha's identification of it. In 1925, Samuel T. Orton determined that there was a syndrome unrelated to brain damage that made learning to read difficult. Since then the researches on dyslexia develops very fast and have yielded lots of fruits in alphabetic language in the perspectives of cognition, neurobiology and genetics. In contrast to the alphabetic scripts, Chinese language is characterized as a logographic writing system with deep orthography. The correspondence between orthography and phonology in Chinese is more arbitrary than in the writing systems with shallow orthographies, like Serbo-Croatian or English. Due to the differences between Chinese language and alphabetic languages, whether the findings in alphabetic languages are coincident in Chinese remains a question. We hypothesize that researches in L1 Chinese learners would reveal that DD causes in Chinese learners are not coincident with that in alphabetic languages.

\section{Acknowledgement of Chinese Dyslexia}

Early researches on dyslexia have focused mainly on the English language, even some researcher claimed that there are few dyslexic children in oriental languages such as Chinese in which the smallest written units are characters representing monosyllabic morphemes (units of language that convey meaning). In 1982, a reading test was constructed in English, Japanese, and Chinese with large samples of children in Japan, Taiwan, and the United States. Strong evidence was found that reading disabilities exist among Chinese and Japanese as well as among American children, which doubts the crucial significance of orthography as the major factor determining the incidence of reading disabilities across cultures [3]. In the following years, Su et al. (1984) found out there exists the dyslexic children in Taiwan schools. Shu et al. (2000) found that Chinese children have difficulties in reading by using survey and case studies [4] and Zhang Chengfen et al. (1998) claimed that detection rate of Children with dyslexia is $7.96 \%$ and $4.55 \%$ depending on the different survey methods in Shandong Province [5]. Since then, many researchers (Meng, Sui, and Li, etc.) began to study DD in Chinese dyslexia and more and more studies show that the presence of dyslexia in Chinese children is an unquestionable fact, and its incidence is very close to that of English.

\section{Dyslexic Theories in Alphabetic Languages}

On the basis of studies, researchers gradually propose many theories in the causes of dyslexia in alphabetic 
language from the perspectives of cognition, neurobiology and genetics, which should be viewed as theories trying to explain the underlying causes of a similar set of symptoms from a variety of research perspectives and background.

\subsection{Cognitive Explanations}

Over 30 years of research indicates that one primary cause of dyslexia is poorly developed phonological awareness and many theories have been proposed to explain the cognitive and behavioral manifestations of dyslexia. Each of these theories appears to be different but sometimes there is an overlaps.

\subsubsection{Phonological Deficit Hypothesis}

The Hypothesis, which is first proposed in the 1970's by Donald Shankweiler and his colleague Isabelle Liberman, is a prevalent cognitive-level explanation for the cause of dyslexia and has been the leading explanation of difficulties in reading abilities for several decades. The basic hypothesis is reading failure or dyslexia stems from a functional or structural deficit in left hemispheric brain areas associated with processing the sounds of language. Some researchers (Galaburda, Frith, and Paulesu, etc.) have studied the structure and function of neural pathways in the language areas of the brain. Others (Stein, Richardson, and Evans, etc.) have focused on the perception of short or rapidly varying sounds of language, positing that the core deficit is one of timing rather than of overall function [6]. The theory is based around speech sounds, and postulates that dyslexic individuals have difficulties in representing, storing and/or retrieving these sounds. It proposes that people with dyslexia have a specific sound manipulation impairment, which affects their auditory memory, word recall, and sound association skills when processing speech. The phonological theory explains a reading impairment. The relationship between the graphic letter symbols and speech sounds which they represent when using an alphabetic writing system which requires learning the grapheme/phoneme correspondence [7].

\subsubsection{Double-deficit Hypothesis}

Wolf \& Bowers (1999) proposed an alternative conceptualization of dyslexia-the double-deficit hypothesis (DDH) - according to which there exist two independent underlying sources of dyslexic difficulties, namely, the phonological core deficit and naming speed impairment [8]. Phonological deficits and deficits in rapid automatized naming (RAN) speed are largely independent sources of reading difficulties. According to the DDH, the majority of poor readers can be classified into three groups: two with a single deficit in either phonological awareness or RAN and one with a double deficit in both phonological awareness and RAN. Because phonological awareness and RAN deficits are assumed to have independent negative effects on reading, children in the double-deficit group are expected to experience the most severe reading problems. It is a model-driven approach to subtype developmental dyslexia, which asserts that individuals with phonologically based deficits and/or naming speed deficits constitute separate classes of dyslexic readers. Within the model, four subtypes are classified, according to the presence or absence of phonological deficits and naming speed deficits (NSDs), with the most impaired subtype exhibiting both deficits, and the smallest subtype characterized by neither. Cirino et al. (2005) extend the applicability of it beyond children, supporting the fact that both phonological processing and visual naming speed seem likely to predict reading deficits in adults [9].

\subsection{Neurobiological Findings}

The researchers (Ramus, Brown, and Stein, etc.) have examined the neurobiological underpinning of reading and dyslexia by CT, functional brain image, and functional magnetic resonance image etc. and proposed some theories. The cerebellar theory and magnocellular theory are the two theories that mostly accepted by researchers.

\subsubsection{The Cerebellar Theory}

The Theory claims that the cerebellum of people with dyslexia is mildly dysfunctional and in cognitive difficulties, including balance, motor skill, phonological skill and rapid processing. First, the cerebellum plays a role in motor control and therefore in speech articulation. It is postulated that retarded or dysfunctional articulation would lead to deficient phonological representations. Secondly, the cerebellum plays a role in the automatization of over-learned tasks, such as driving, typing and reading. A weak capacity to automatize would affect, among other things, the learning of graphemephoneme correspondences [10]. Brain imaging studies have also shown anatomical, metabolic and activation differences in the cerebellum of dyslexics [11].

\subsubsection{The Magnocellular Theory}

The Theory postulates that the magnocellular dysfunction is not restricted to the visual pathways but is generalized to all modalities (visual and auditory as well as tactile) and the deficit lies in the perception of short or rapidly varying sounds or difficulty processing the letters and words on a page of text. Evidence to support this theory includes differences in the dyslexic brain anatomy in both visual and auditory magnocellular pathways [12].

\subsection{Genetics}

Dyslexia is a heritable disorder and family history is an important risk factor, with 23-65\% of children with family history of dyslexic also found to be dyslexic [13]. There is strong evidence (affected parent) for a genetic contribution to the disorder. When children of consanguineous marriages were examined for their reading disabilities, the rate of reading disabilities is higher in children of first cousin parents than that of second-cousin or unrelated parents. This suggests a genetic basis of reading disabilities [14].

The main advance in the genetics of dyslexia has been the identification of six candidate genes (DYX1C1 in the DYX1 locus on chromosome 15q21; DCDC2 and KIAA0319 in the DYX2 locus on chromosome 6p21; C2Orf3 and MRPL19 in the DYX3 locus on chromosome 2p16-p15; and ROBO1 in the DYX5 locus on chromosome 3p12-q12) and studies of their role in brain development.114 Furthermore, Poelmans et al. identified a further four possible areas (PCNT, DIP2A, S100B and PRMT2) on chromosome region21q22.3 [15]. 


\section{Findings in Chinese Dyslexics}

Chinese language is one of the oldest languages in the world and spoken by the largest population in the world. It is a morph-syllabic writing system, where the basic graphic unit, the Chinese character, represents a morpheme as well as a syllable, and characters can be segmented using orthographic, morphological and syllabic information [16]. Chinese characters map onto syllables rather than phonemes. The homophonic characters can be visually dissimilar and the orthographically similar characters can have different pronunciations. Given these overarching differences between Chinese and alphabetic scripts, the Chinese researchers' work provide some new findings of which some are in coincidence with existing theories and some are new.

\subsection{Cognitive Findings}

Li \& Shu (2009) tested 41 dyslexic Chinese children and 41 normal readers from Grade-5 and Grade-6 in a battery of linguistic and non-linguistic cognitive tasks and reading tasks. The results indicated that the dyslexic children performed significantly worse than the normal children in all linguistic cognitive tasks and literacy skill tests [17]. Zou et al (2009) claim that dyslexic children were slower to recognize target characters than normal readers, and did not exhibit significant prime effects in graphic and phonological primer condition [18]. These results suggested that dyslexic children exhibited general deficits in phonological and graphic processing, and presented a different pattern in character recognition.

In Zheng et al's research (2007), three tests (phonological skill naming speed and word form processing tests) were designed to investigate the linguistic capacity of Chinese dyslexic children and conclusions are that Chinese dyslexic children have deficiency in the linguistic capacity of phonological awareness and naming speed ability [19]. Sui et al (2012) found out that there are deficiencies in the morphological awareness in developmental dyslexia; the backwardness of morphological differentiation and usage illustrates that deficiency in morphological awareness is the main cause of developmental dyslexia in Chinese [20] . Sui et al (2010) suggest that the defects of those children with Chinese-language development dyslexia in morphemic understanding, phonological awareness and orthographic awareness are supported [21].

Li et al (2009) find that there is a detectable disability for the Chinese dyslexic children in the visuospatial cognitive processing: their saccade amplitude and mean saccade distance are shorter, which may be interpreted as specific for their reading disability [22]. Siok et al (2009) claims their study demonstrates that developmental dyslexia in Chinese is typically characterized by the coexistence of visuospatial and phonological disorders in a dyslexic child [23]. This pattern of behavioral and pathophysiological profiles is different from that in English dyslexia, which is generally associated with a core phonological deficit in the absence of abnormal visual processing. Meng et al (2011) conclude that reading development in Chinese depends to a certain extent on the development of dynamic visual perception and its underlying neural pathway and that the impact of visual development can be specifically related to orthographic processing in reading Chinese [24].

Dyslexic children possessed processing deficits in these two accesses: the first one was from grapheme to meaning ;the second one was from grapheme-phonology to meaning [25]. Dong et al (2012) report that dyslexic children were effectively distinguished from normal children with tasks of phonological awareness, morphological awareness, orthographic awareness and rapid naming [26] and they come to the conclusion: multiple reading-related cognitive deficits may be the main cause for Chinese developmental dyslexia, which importantly contributed to the diagnosis and intervention of Chinese dyslexic children. Mo et al (2013) adapted a cluster random sampling method was used to select 58 children from Grade 3-6 in a primary school and the results shows that there were five cognitive deficits existing in dyslexia children including morphological awareness, naming speeding, working memory, speech short-term memory and phonological awareness. The phonological awareness was the most important [27].

\subsection{Neurobiological Findings}

Siok (2004) proposes that functional disruption of the left middle frontal gyrus is associated with impaired reading of the Chinese language (a logographic rather than alphabetic writing system) [28]. Reading impairment in Chinese is manifested by two deficits: one relating to the conversion of graphic form (orthography) to syllable, and the other concerning orthography- to-semantics mapping. Both of these processes are critically mediated by the left middle frontal gyrus, which functions as a centre for fluent Chinese reading 9-11 that coordinates and integrates various information about written characters in verbal and spatial working memory. This finding provides an insight into the fundamental pathophysiology of dyslexia by suggesting that rather than having a universal origin, the biological abnormality of impaired reading is dependent on culture. Later, Siok (2008) finds that different structural and functional abnormalities in dyslexic readers of Chinese [29]. Compared with normally developing controls, children with impaired reading in logographic Chinese exhibited reduced gray matter volume in a left middle frontal gyrus region previously shown to be important for Chinese reading and writing. The results suggest that the structural and functional basis for dyslexia varies between alphabetic and non-alphabetic languages.

$\mathrm{Li}$ et al (2013) compared morphological processing in a $\mathrm{RD}$ group (11-13 years old) to an age-matched typically developing (TD) group by using functional magnetic resonance imaging (fMRI) [30]. A smaller incongruency effect was found in left dorsal posterior (BA9) and ventral anterior (BA47) inferior frontal gyrus (IFG) in the RD compared to the $\mathrm{TD}$, suggesting that the $\mathrm{RD}$ is less sensitive to morphological information. Moreover, brain activation in the IFG for the incongruency effect in the semantic task was negatively correlated with reading skill for the RD group only, suggesting that higher skill children with RD may rely on a compensatory wholeword strategy by ignoring the morphemic information. Kuo (2003) reports the activation pattern of reading infrequently encountered characters reflects a more demanding processing procedure of retrieving, formulating, and coordinating the phonological output [31]. 
Access to the lexical route may benefit the reading of high-frequency characters. By uncovering the differential brain responses in reading Chinese characters of different occurrence frequencies, not only has a substantial overlap between functional neuroanatomy of reading Chinese and alphabetical languages been demonstrated, but also features permitting the separation of language-specific content from universal mechanisms. And Kuo (2004) points out that the ventral part of the left inferior frontal cortex responds specifically to the character stimuli, suggesting a general lexical processing role for this region for linguistic material [32]. The stronger activation of the dorsal visual stream by Chinese homophone judgment pinpoints a tight coupling between phonological representation of Chinese characters and corresponding orthographic percepts.

\subsection{Genetic Findings}

Cadmon KP Lim et al (2012) find that genotyped eight single nucleotide polymorphisms (SNPs) from three hundred and ninety three individuals from 131 Chinese families with two which have been reported in the literature and six tag SNPs at DYX1C1 and the findings suggest that DYX1C1 is associated with dyslexia in people of Chinese ethnicity in Hong Kong [33]. And Yuping Zhang et al (2012) investigated the contribution of DYX1C1 to reading, with a focus on orthographic skills, in a sample of 284 unrelated Chinese children aged 5 to 11 years who were participating in the Chinese Longitudinal Study of Reading Development and suggest that DYX1C1 influences reading development in the general Chinese population and supports a universal effect of this gene [34].

\section{Conclusion}

By reviewing the previous studies in L1 Chinese learners, we find out that although DD in Chinese language have been studied from perspectives of cognition, neurobiology and genetics, which is coincident with those of alphabetic languages, there exits differences in some minor parts. The following table shows the commons and differences between Chinese language and alphabetic languages.

Table 1. Cognitive Perspective in Alphabetic Languages and Chinese

\begin{tabular}{|c|c|}
\hline Commons & $\begin{array}{c}\text { Difficulties with the accuracy and speed of character or word recognition; } \\
\text { Phonological and naming-speed deficits are common. }\end{array}$ \\
\hline Differences & $\begin{array}{c}\text { Orthographic and morphological deficits seem to be important features specific to Chinese; } \\
\text { Most Chinese children with dyslexia tend to have more than one kind of cognitive deficit. }\end{array}$ \\
\hline
\end{tabular}

Table 2. Neurobiological Perspective in Alphabetic Languages and Chinese

\begin{tabular}{|c|c|}
\hline \multirow{2}{*}{ Commons } & $\begin{array}{c}\text { Mildly dysfunctional and in cognitive difficulties; } \\
\text { A weak capacity to automatize would affectthe learning of grapheme-phoneme correspondences; } \\
\text { Differences in the dyslexic brain anatomy in both visual and auditory magnocellular pathways. }\end{array}$ \\
\hline \multirow{2}{*}{ Differences } & $\begin{array}{c}\text { Functional disruption of the left middle frontal gyrus is associated with impaired reading of the Chinese language; } \\
\text { The left inferior frontal cortex responds specifically to the Chinese character stimuli; }\end{array}$ \\
Children with impaired reading in Chinese exhibited reduced gray matter volume in a left middle frontal gyrus region.
\end{tabular}

Table 3. Genetic Perspective in Alphabetic Languages and Chinese

\begin{tabular}{|c|c|}
\hline Common & Candidate gene: DYX1C1 \\
\hline Differences & $\begin{array}{r}\text { More candidate genes for alphabetic languages: DCDC2 and KIAAO319 in the DYX2 locus on chromosome 6p21; C2Orf3 and MRPL19 } \\
\text { in the DYX3 locus on chromosome 2p16-p15; and ROBO1 in the DYX5 locus on chromosome 3p12-q12 }\end{array}$ \\
\hline
\end{tabular}

From the tables above, we can easily conclude that DD in Chinese learners has its own characteristics, though it shares some commons of those in alphabetic languages findings, owing to the differences in the morph-syllabic languages and alphabetic languages.

In the future DD studies in L1 Chinese learners, the researchers may find more fills to gap the differences between the two language systems. There remain lots of topics to be studied. In cognitive aspect, besides the above finding, whether other differences need to be found out? In the neurobiological aspect, the findings shows left middle frontal gyrus region is associated with L1 Chinese learners, and can we try to help Chinese dyslexics from this aspect? In the genetic studies, whether the second candidate gene for L1 Chinese learner exists? Or, whether DYX1C1 is the only gene for L1 Chinese learners?

Moreover, there are no studies in the L2 Chinese learner of English dyslexics (or native speakers of other alphabetic languages who are diagnosed as dyslexic), what will happen when an English dyslexic learns Chinese as L2? Does he meet the same reading problems in L2 Chinese as well as in L1 English? Do the findings of him can also be explained in the framework of dyslexic theories?
A lot of work needs to be done in the future in the field of dyslexia.

\section{Acknowledgement}

The paper was prepared with support of Science Foundation of Beijing Language and Culture University (supported by "the Fundamental Research Funds for the Central Universities”) (Approval No. 13 YBG02).

\section{References}

[1] Barkur S.Shastry, "Developmental dyslexia: an update”, Journal of Human Genetics, 52: 104-109, 2007.

[2] Margaret J. Snowling, "Early identification and interventions for dyslexia:a contemporary view”, Journal of Research in Special Educational Needs, 13 (1): 7-14, 2013.

[3] Harold W. Stevenson, James W. Stigler, G. William Lucker, Shinying Lee, Chen-chin Hsu and Seiro Kitamura, "Reading Disabilities: The Case of Chinese, Japanese, and English”, Child Development, 53 (5): 1164-1181, 1982.

[4] Shu Hua and Meng Xiangzhi, “A preliminary study of reading disability of Chinese speaking children-- Statistical data of children with reading disability”, Applied Linguistics, 3: 63-69, Aug, 2000. 
[5] Zhang Chengfen, Zhang Jinghuan, Chang Shumin, Zhou Jing, “A Study of Cognitive Profiles of Chinese Learners' Reading Disability”, Acta Psychologica Sinica, 30 (1): 50-56, 1998.

[6] Ramus, Franck, "Dyslexia: Talk of two theories", Nature, 412 (6845): 393-395, 2001.

[7] Ramus F, Rosen S, Dakin SC. "Theories of developmental dyslexia: insights from a multiple case study of dyslexic adults", Brain, 126 (4): 841-65, April. 2003.

[8] Wolf, M. and Bowers, P.G, "The double-deficit hypothesis for the developmental dyslexia”, Journal of Educational Psychology, 91: 415-438, 1999.

[9] Cirino, P.T., Israelian, M.K., Morris, M.K. and Morris, R.D. "Evaluation of the double-deficit hypothesis in college students referred for learning difficulties”, Journal of Learning Disabilities, 38 (1): 29-43, 2005.

[10] Ramus, R.; Rosen, S.; Dakin, S.; Day, B.; Castellote, J.; White, S.; Frith, U. "Theories of Developmental Dyslexia: Insights from a Multiple Case Study of Dyslexic Adults”, Brain, 126: 841-865, 2003.

[11] Brown, E. E.; Eliez, S.; Menon, V.; Rumsey, J. M.; White, C. D.; Reiss, A. L. "Preliminary Evidence of Widespread Morphological Variations of the Brain in Dyslexia”, Neurology, 56: 781-783, 2001.

[12] Stein, J. “The Magnocellular Theory of Dyslexia”, Dyslexia, 7: 12-36, 2001.

[13] Shaywitz S, Shaywitzx BA. "The science of reading and dyslexia”, Journal of American Association for Pediatric Ophthalmology and Strabismus, 7: 158-166, 2003.

[14] Abu-Rabia S, Maroun L, "The effect of consanguineous marriage on reading disability in the Arab community”, Dyslexia, 11: 1-21, 2005.

[15] Poelmans G, Engelen JJ, Van Lent-Albrechts J et al, "Identification of novel dyslexia candidate genes through the analysis of a chromosomal deletion”, American Journal of Medical Genetics, 150B (1): 140-7, 2009.

[16] Shu, H., \& Anderson, R. C. "Role of radical awareness in the character and word acquisition of Chinese children”, Reading Research Quarterly, 32:78-89, 1997.

[17] Li Hong, Shu Hua, “The Linguistic Cognitive Deficiency of Developmental Dyslexic Children”, Psychological Science, 32 (2) : 301- 303, 2009.

[18] Yuliang Zou, Jing Wang, Hanrong Wu, "Priming effects in Chinese character recognition for Chinese children with developmental dyslexia”, Neural Regeneration Research, 4 (6):474-480, 2009.

[19] Zheng Shuhui, Huang Xu, Jing Jin, "Deficiency in the linguistic capacity in the Chinese dyslexic children”, Chinese Mental Health Journal, 21 (2): 75-78, 2007.

[20] Sui Xue, Liu Wen, Weng Xuchu, "Morphological Awareness and Usage in Chinese Primary School Children with Developmental Dyslexia”, Journal of Liaoning Normal University (Social Science Edition,) 35 (1):54-59, 2012.

[21] Sui Xue, Jiang Na, Qian Li, “A Research on Eye-movement in Word Reading by Children with Chinese-language Development
Dyslexia”, Chinese Journal of Special Education, 117 (3): 63-67, 2010.

[22] LI Xiuhong, JING Jin, ZOU Xiaobing, HUANG Xu, JIN Yu, WANG Qing-xiong, CHEN Xuebin, YANG Binrang and YANG Siyuan, "Picture perception in Chinese dyslexic children: an eyemovement study”, Chinese Medical Journal, 122 (3): 267-271, 2009.

[23] Wai Ting Siok, John A. Spinks, Zhen Jin, Li Hai Tan, "Developmental dyslexia is characterized by the co-existence of visuospatial and phonological disorders in Chinese children”, Current Biology, 19 (19): 890-892, 2009.

[24] Xiangzhi Meng, Alice Chenglai, Biao Zeng, John F. Stein, Xiaolin Zhou, "Dynamic visual perception and reading development in Chinese school children”, Annals of Dyslexia, 61: 161-176, 2011.

[25] Song Rangrand, Wu Hanrong, "Cognitive chararteristics for Chinese characters in children with dyslexia”, Chinese Journal of Public Health, 24 (2): 181-183, 2008.

[26] Dong Qiong, Li Hong, Wu Xinchun, Pan Jiner, Zhang Yuping, Ruan Shifang, "Multiple Reading-related Cognitive Deficits in Chinese Developmental Dyslexia”, Chinese Journal of Clinical Psychology, 20 (6): 798-801, 2012.

[27] Mo Shengnan, Wang Bo, He Zhen, SUN Zhao, Shao Shanshan, Song Ranran, "Study on early cognitive deficits among children with dyslexia”, Chinese Journal of Health Education, 29 (4): 316319, April 2013.

[28] Wai Ting Siok, Charles A. Perfetti, Zhen Jin, Li Hai Tan, "Biological abnormality of impaired reading is constrained by culture”, Nature, 431: 71-76, 2004.

[29] Wai Ting Siok, Zhendong Niu, Zhen Jin, Charles A. Perfetti_, and Li Hai Tan, "A structural-functional basis for dyslexia in the cortex of Chinese readers”, PNAS, 105 (14): 5561-5566, 2008.

[30] Li Liu, Ran Tao, Wenjing Wang, Wenping You, Danling Peng, James R. Booth, "Chinese dyslexics show neural differences in morphological processing”, Developmental Cognitive Neuroscience, 6 (2013): 40- 50, 2013

[31] Wen-Jui Kuo, Tzu-Chen Yeh, Chia-Ying Lee, Yu-Te Wu, ChiCher Chou, Low-Tone Ho, Daisy L. Hung, Ovid J.L. Tzeng, and Jen-Chuen Hsieh, "Frequency effects of Chinese character processing in the brain: an event-related fMRI study", Neuro Image, 18 (2003): 720-730, 2003.

[32] Wen-Jui Kuo, Tzu-Chen Yeh, Jun-Ren Lee, Li-Fen Chen,Po-Lei Lee, Shyan-Shiou Chen, Low-Tone Ho, Daisy L. Hung,Ovid J.-L. Tzeng, and Jen-Chuen Hsieh, "Orthographic and phonological processing of Chinese characters: an fMRI study”, NeuroImage, 21 (2004): 1721-1731, 2004.

[33] Cadmon KP Lim, Connie SH Ho, Crystal HN Chou and Mary MY Waye, "Association of the rs3743205 variant of DYX1C1 with dyslexia in Chinese children”, Behavioral and Brain Functions, 2011 (7): 16, 2011.

[34] Yuping Zhang, Jun Li, Twila Tardif, Margit Burmeister, Sandra M. Villafuerte, Catherine McBride-Chang, Hong Li, Bingjie Shi, Weilan Liang, Zhixiang Zhang, Hua Shu, "Association of the DYX1C1 Dyslexia Susceptibility Gene with Orthography in the Chinese Population ”, Plos one, 7 (9): e 42969, 2012. 